Pacific Journal of Mathematic 


\title{
SOME RESULTS ON NORMALITY OF A GRADED RING
}

\author{
WEI-EIHN KUAN
}

\begin{abstract}
Let $R=\bigoplus_{i \geqq 0} R_{i}$ be a graded domain and let $p$ be a homogeneous prime ideal in $R$. Let $R_{\mathrm{p}}$ be be the localization of $R$ at $\mathbf{p}$ and $R_{(\mathbf{p})}=\left\{r_{i} / s_{i} \mid r_{i} s_{i} \in R_{i}\right.$ and $\left.s_{i} \notin \mathbf{p}\right\}$. If $R_{1} \cap(R-\mathbf{p}) \neq \varnothing$, then $R_{\mathrm{p}}$ is a localization of a transcendental extension of $R_{(\mathrm{p})}$. Thus $R_{\mathrm{p}}$ is normal (regular) if and only if $R_{(\mathrm{p})}$ is normal (regular). Let $\operatorname{Proj}(R)=\{\mathbf{p} \mid \mathbf{p}$ is a homogeneous prime ideal and $\left.p \varsubsetneqq \bigoplus_{i>0} R_{i}\right\}$. Under certain conditions a Noetherian graded domain $R$ is normal if $R_{(\mathbf{p})}$, is normal for each $\mathbf{p} \in \operatorname{Proj}(R)$. If $R=\bigoplus_{i \geqq 0} R_{i}$ is reduced and $F_{0}=\left\{r_{i} / u_{i} \mid r_{i}, u_{i} \in R_{i}\right.$ and $u_{i} \in U$ where $U$ is the set of all nonzero divisors $\}$ is Noetherian, then the integral closure of $R$ in the total quotient ring of $R$ is also graded.
\end{abstract}

1. Introduction. Let $R=\bigoplus_{i \geqq 0} R_{i}$ be a graded integral domain. Let $\operatorname{Spec}(R)$ be the set of all prime ideals in $R$. Let $R_{+}=\bigoplus_{i>0} R_{i}$. $R_{+}$is an ideal in $R$. An ideal $\mathfrak{A}$ in $R$ is said to be irrelevant if $R_{+} \subset \sqrt{\mathfrak{A}}$, the radical of $\mathfrak{A}$. Let $\operatorname{Proj}(R)=\left\{\mathbf{p} \in \operatorname{Spec}(R) \mid \mathbf{p} \subset R_{+}\right.$is homogeneous and nonirrelevant $\}$. For each $\mathbf{p} \in \operatorname{Spec}(R)$, let $R_{\mathrm{p}}=\{r / s \mid, s \in R$ and $s \notin \mathbf{p}\}$, and for each homogeneous prime ideal $\mathbf{p}$, let $R_{(\mathbf{p})}=\left\{r_{i} / s_{i} \mid r_{i}, s_{i} \in R_{i}\right.$ and $\left.s_{i} \notin \mathbf{p}\right\}$. (Note: $R_{(\mathbf{p})}$ in [1] is defined for $\mathbf{p} \in \operatorname{Proj}(R)$ only.) According to the terminology of Seidenberg [9], $R_{\mathrm{p}}$ is called the arithmetical local ring of $R$ at $\mathbf{p}$ and $R_{(\mathbf{p})}$ the geometrical local ring of $R$ at $\mathbf{p}$. I prove that if $R_{I} \cap(R-\mathbf{p}) \neq \varnothing$ then $R_{\mathrm{p}}$ is the ring of quotients of a transcendental extension of $R_{(\mathrm{p})}$ relative to a multiplicative set, $R_{\mathrm{p}}$ is normal (regular) if and only if $R_{(\mathrm{p})}$ is normal (regular); see Theorem 2. In the case of an irreducible projective variety $V$ over a field $k$ in a projective $n$-space $P_{k}^{n}$, $V / k$ is normal if the geometrical local ring of $V$ at each $\mathbf{p} \in V, \mathfrak{D}_{k}^{v}(\mathbf{p})$ is integrally closed. $V$ is arithmetically normal if the ring of strictly homogeneous coordinates $k[V]$ is integrally closed. The latter implies the former. For the converse, various cohomological criteria are developed; see [3], [8], [9]. I attempt to study the normality of a graded domain $R$ if $R_{(\mathbf{p})}$ is normal for every $\mathbf{p} \in \operatorname{Proj}(R)$. In this paper, I also obtain the following theorem: Let $R$ be a Noetherian graded domain, say $R=R_{0}\left[x_{1}, \cdots, x_{n}\right]$ and $x_{1}, \cdots, x_{n}$ are of homogeneous degree 1 . Assume that $R_{0}$ contains a field $k$ over which $R_{0}$ and $k\left(x_{1}, \cdots, x_{n}\right)$ are linearly disjoint and separable. Let $\mathfrak{B}$ be the kernel of the canonical map from the polynomial ring $R_{0}\left[X_{1}, \cdots, X_{n}\right]$. Then $R$ is normal if $R_{0}$ is normal, $R_{(\mathrm{p})}$ is normal for every $\mathbf{p} \in \operatorname{Proj}(R)$ and coh.d. $\mathfrak{B} \cdot K\left[X_{1}, \cdots, X_{n}\right]<n-1$, where $K$ is the quotient field of $R_{\boldsymbol{0}}$. 
In the $\S 4$, we prove that under certain conditions on a graded ring $R$ (not necessarily integral domain) the integral closure $\bar{R}$ of $R$ in the total quotient ring of $R$ is also graded; see Theorem 6 .

Our references on the elementary well known facts about graded rings can be found in [1] and [10].

I would like to thank Professor A. Seidenberg for many valuable discussions and suggestions during preparation of the research, while I was on sabbatical leave visiting Berkeley.

I would like also to thank the referee for his comments.

2. Normality and regularity of local domains. Let $R$ be a commutative ring with identity 1 . Let $\mathbf{p}$ be a prime ideal in $R$. By height of $\mathbf{p}$, we mean the supremum of the length of chains of prime ideals $\mathbf{p}_{\boldsymbol{o}} \supsetneqq \mathbf{p}_{1} \supsetneqq \mathbf{p}_{2} \supsetneqq \cdots \mathbf{p}_{n}$ with $\mathbf{p}_{\boldsymbol{o}}=\mathbf{p}$ and denote it by $h t(\mathbf{p})$. Let $R=\bigoplus_{i \geqq 0} R_{i}$ be a graded integral domain. Let $K$ be the quotient field of $R$. We say that $R$ is integrally closed if $R$ is integrally closed in $K$. Let $K_{q}=\left\{f_{i} / g_{j} \mid i-j=q ; f_{i} \in R_{i}, g_{j} \in R_{j}\right\} . K_{o}$ is a field, $\Sigma_{q \in Z} K_{q}$ is a subring of $K$ and the sum is direct, where $Z$ stands for the set of integers. Elements in $K_{q}$ are known as homogeneous elements of $K$ of degree $q$. The following theorem was originally proved in [9] for projective varieties. We observe that the same holds true for non-Noetherian graded domain also.

THEOREM 1. Let $R=\bigoplus_{i \geqq 0} R_{i}$ be a graded domain. Let $\mathbf{p} \in \operatorname{Spec}(R)$ be nonhomogeneous. If $h t(\mathbf{p})=1$ then $R_{\mathbf{p}}$ is integrally closed.

Proof. Let $\mathbf{p}^{*}$ be the ideal generated by all the homogeneous elements of p. By [10, Lemma 3, p. 153] $\mathbf{p}^{*}$ is a prime ideal and $\mathbf{p} ¥ \mathbf{p}^{*} \geqq 0$. Since $h t(\mathbf{p})=1, \mathbf{p}^{*}=0$. Therefore $\mathbf{p}$ contains no homogeneous element. Thus every nonzero homogeneous element $u$ is in $R-\mathbf{p}$. It follows therefore $\bigoplus_{q \in Z} K_{q} \subset R_{\mathrm{p}}$. Let $f \in K$ be integral over $R_{\mathrm{p}}$. Then there exists $h \in R-\mathbf{p}$ such that $f h$ is integral over $R$. It follows from [10, Theorem 11, p. 157] that each of the homogeneous components is integral over $R$. By the preceeding, each homogeneous component of $f \cdot h$ is in $R_{\mathrm{p}}$. Therefore $f \cdot h \in R_{\mathrm{p}}$ and $f \in R_{\mathrm{p}}$. Thus $R_{\mathrm{p}}$ is integrally closed.

Let $y \in K_{1}$ be any nonzero element. If $\xi \in K_{q}$, then $\xi / y^{q} \in K_{0}$. Moreover $R \subset K_{0}[y], K=K_{0}(y), y$ is transcendental over $K_{0}, K_{q}=K_{0} y^{q}$ and $\bigoplus_{q \in Z} K_{q}=K_{o}[y, 1 / y]$. We have the following theorem.

THEOREM 2. ${ }^{\dagger}$ Let $R=\bigoplus_{i \geqq 0} R_{i}$ with that $R_{1} \neq 0$. Let $\mathbf{p}$ be $a$ homogeneous prime ideal such that there exists an element $r_{1} \in R_{1}-\mathbf{p}$. Then

${ }^{+}$Professor A. Seidenberg remarks that the present Theorem 2 strengthens Lemma 2 of [9; $p$. 618] and corrects its proof. 
(a) $K_{0}$ is the quotient field of $R_{(\mathrm{p})}$ and $K_{0} \cap R_{\mathrm{p}}=R_{(\mathrm{p})}$. in $K$.

(b) $R_{(\mathrm{p})}$ is integrally closed in $K_{0}$ implies that $R_{(\mathrm{p})}$ is integrally closed

(c) $R_{\mathrm{p}}=\left(R_{(\mathrm{p})}\left[r_{1}\right]\right)_{s}$, where $S=R-\mathbf{p} ; r_{1}$ is transcendental over $R_{(\mathrm{p})}$. in $K_{0}$.

(d) $R_{\mathrm{p}}$ is integrally closed in $K$ if and only if $R_{(\mathrm{p})}$ is integrally closed

(e) $R_{(\mathrm{p})}$ is regular if and only if $R_{\mathrm{p}}$ is regular.

Proof. By definition $R_{(\mathbf{p})} \subset K_{0}$. Let $x \in K_{0}, \quad x=f_{i} / g_{i}$ for some $f_{i}, g_{i} \in R_{i}$ and $g_{i} \neq 0$. Then $x=f_{i} / g_{i}=\left(f_{i} / r_{1}^{i}\right) /\left(g_{k} / r_{1}^{i}\right)$, since $f_{k} / r_{1}^{i}$ and $f_{i} / r_{1}^{i}$ are both in $R_{(\mathrm{p})}$. Therefore $x$ is in the quotient field of $R_{(\mathrm{p})}$. Thus $K_{0}$ is the quotient field of $R_{(p)}$. For the second part of (a) we need only to prove that $K_{0} \cap R_{\mathrm{p}} \subset R_{(\mathrm{p})}$. Let $x \in K_{0} \cap R_{\mathrm{p}}$. Then $x=f_{i} / g_{i}$ for some $f_{i}$, $g_{i} \in R_{i}$ with $g_{i} \neq 0$. On the other hand $x=\left(r_{j}+r_{j+1}+\cdots+r_{j+m}\right) /$ $\left(s_{l}+s_{l+1}+\cdots+s_{l+m}\right)$ with $s_{l}+s_{l+1}+\cdots+s_{l+m} \notin \mathbf{p}$. Then there exists an index $\quad l+t$ such that $s_{l+t} \notin \mathbf{p} . \quad f_{i} \cdot\left(s_{l}+s_{l+1}+\cdots+s_{l+m}\right)=$ $g_{i}\left(r_{j}+r_{j+1}+\cdots+r_{j+k}\right)$ implies that $l=j, m=k$ and $f_{i} \cdot s_{l+t}=g_{i} \cdot r_{l+t \cdot}$ Thus $x=f_{i} / g_{i}=r_{l+t} / s_{l+t}$ i.e. $x \in R_{(\mathrm{p})}$. Therefore $K_{0} \cap R_{\mathrm{p}}=R_{(\mathrm{p})}$.

(b) If $R_{(\mathrm{p})}$ is integrally closed in $K_{0}$, then, since $K=K_{0}\left(r_{1}\right)$ and $r_{1}$ is transcendental over $K_{0}$ as noted in the preceeding, $K_{0}$ is algebraically closed in $K$ and $R_{(\mathbf{p})}$ is thus integrally closed in $K$.

(c) As noted in (b), $r_{1}$ is transcendental over $R_{(\mathrm{p})}$. Let $f \in R$ be an element. Then $f=f_{r}+f_{r+1}+\cdots+f_{n}$ where $f_{i} \in R_{i}$ for some nonnegative integers $r$ and $n$. But $f=\left(f_{r} / r_{1}^{r}\right) r_{1}^{r}+\left(f_{r+1} / r_{1}^{r+1}\right) r_{1}^{r+1}+\cdots+\left(f_{n} / r_{1}^{n}\right) r_{1}^{n} \in R_{(\mathrm{p})}\left[r_{1}\right]$. Therefore $R \subset R_{(\mathrm{p})}\left[r_{1}\right]$. Thus $S=R-\mathbf{p}$ is a multiplicative set in $R_{(\mathrm{p})}\left[r_{1}\right]$. Now let $f / g \in R_{\mathrm{p}}, g \in R-\mathbf{p}$. Then for some nonnegative integer $t$ and $m$,

$$
\frac{f}{g}=\frac{f_{t}}{g}+\cdots+\frac{f_{m}}{g}=\frac{1}{g}\left(\left(\frac{f_{t}}{r^{t}}\right) r_{1}^{t}+\left(\frac{f_{t+1}}{r^{t+1}}\right) r_{1}^{t+1}+\cdots+\left(\frac{f_{m}}{r_{1}^{m}}\right) r_{1}^{m}\right) .
$$

Therefore $f / g \in\left(R_{(\mathbf{p})}\left[r_{1}\right]\right)_{s}$ i.e. $R_{\mathrm{p}} \subset\left[R_{(\mathrm{p})}\left[r_{1}\right]\right)_{s}$. The other inclusion is obvious. Thus $R_{\mathrm{p}}=\left(R_{(\mathrm{p})}\left[r_{1}\right]\right)_{s}$.

(d) Now, if $R_{(\mathrm{p})}$ is integrally closed in $K$, then clearly $R_{\mathrm{p}}=$ $\left(R_{(\mathbf{p})}\left[r_{1}\right]\right)_{s}$, being a localization of transcendental extension of an integrally closed domain, is integrally closed. Conversely if $R_{\mathrm{p}}$ is integrally closed in $K$, let $f \in K_{0}$ be an integral element over $R_{(\mathrm{p})}$. Then $f \in R_{\mathrm{p}}$. Thus $f \in R_{\mathrm{p}} \cap K_{\mathrm{o}}=R_{(\mathrm{p})}$, and $R_{(\mathrm{p})}$ is integrally closed.

(e) Recall that a ring $A$ is said to be regular if $A_{m}$ is a regular local ring for each maximal ideal $m$ in $A$. It follows from Serre's theorem [5; p. 139] that $A$ is regular if and only if $A_{\mathbf{p}}$ is regular for every $\mathbf{p} \in \operatorname{Spec}(A)$.

If $R_{(\mathrm{p})}$ is a regular local ring, then by [5; Theorem 40, p. 126] the polynomial ring $R_{(\mathrm{p})}\left[r_{1}\right]$ is regular. Since localization of a regular ring is regular therefore $R_{\mathrm{p}}=\left(R_{(\mathrm{p})}\left[r_{1}\right]\right)_{s}$ is a regular local ring. 
Conversely assume that $R_{\mathrm{p}}=\left(R_{(\mathrm{p})}\left[r_{1}\right]\right)_{s}$ is a regular local ring. Since $R_{(\mathrm{p})}\left[r_{1}\right]$ is a polynomial ring over $R_{(\mathrm{p})}$ therefore $R_{(\mathrm{p})}\left[r_{1}\right]$ is $R_{(\mathrm{p})}-$ flat. $\left(R_{(\mathrm{p})}\left[r_{1}\right]\right)_{\mathrm{s}}$ is $R_{(\mathrm{p})}\left[r_{1}\right]$-flat therefore $R_{\mathrm{p}}$ is $R_{(\mathrm{p})}$-flat. Thus $R_{(\mathrm{p})}$ is Noetherian. The inclusion map $R_{(\mathrm{p})} \rightarrow R_{\mathrm{p}}$ is obviously a local homomorphism. Therefore it follows from [1; IV , 17.3.3 (i), p. 48] that $R_{(\mathrm{p})}$ is a regular local ring.

There are graded rings in which there are homogeneous prime ideals $\mathbf{p}$ such that $\mathbf{p} \cap R_{1} \neq R_{1}$. For example: (1) graded rings which are homogeneous coordinate rings of projective varieties. In this case $\mathbf{p} \cap R_{1} \neq R_{1}$ for $\mathbf{p} \in \operatorname{Proj}(R)$. (2) $R=R_{0}\left[R_{1}\right]$, a graded ring generated over $R_{0}$ by $R_{1}$; (3) Let $k[X, Y]$ be a polynomial ring in two indeterminantes over a field $k$. Let $R=k[Y]+(X \cdot Y) \cdot k[X, Y] . R$ has a graded structure $R=R_{0} \oplus R_{1} \oplus R_{2} \oplus \cdots$ with $R_{0}=k, R_{1}=k \cdot Y ; R_{2}=$ $k Y^{2}+k(X \cdot Y), R_{3}=k Y^{3}+k X^{2} Y+k X Y^{2}$, etc. It follows from the observation that $\left(X^{i} \cdot Y^{j}\right)^{2} \in R y$ if $j \geqq 1$ that $\mathbf{p} \cap R_{1}=0$ for every $\mathbf{p} \in \operatorname{Proj}(R)$.

3. Normality of a graded domain. In this section, a graded domain $R$ is normal if it is integrally closed in its field of fractions.

Recall [6; Theorem 8, p. 400]: Let $\mathscr{D}$ and $\mathfrak{D}^{\prime}$ be two normal rings which contain a field $k$. If $\subseteq$ and $\mathfrak{S}^{\prime}$ are separably generated over $k$ and if $\mathfrak{D} \bigotimes_{k} \mathfrak{D}^{\prime}$ is an integral domain, then $\mathfrak{D} \bigotimes_{k} \mathfrak{D}^{\prime}$ is a normal ring.

THEOREM 3. Let $R_{\boldsymbol{b}}$ be a normal integral domain containing a field $k$ such that $R_{0}$ is separable over $k$. Let $R=R_{0}[x]=R_{0}\left[x_{1}, \cdots, x_{n}\right]$ be an integral domain finitely generated over $R_{0}$ as an $R_{0}$-algebra such that the quotient field $K$ of $R_{0}$ and the quotient field $k(x)$ of $k\left[x_{1}, \cdots, x_{n}\right]$ are linearly disjoint over $k$, and $k(x)$ separable over $k$. Then $k[x]$ is normal if and only if $R$ is normal.

Proof. Let $X_{1}, \cdots, X_{n}$ be $n$ indeterminantes over $R_{0}$. Let $\mathfrak{A}$ be the prime ideal in $k[X]=k\left[X_{1}, \cdots, X_{n}\right]$ such that $k\left[x_{1}, \cdots, x_{n}\right] \cong$ $k\left[X_{1}, \cdots, X_{n}\right] / \mathscr{U}$ and let $\mathfrak{B}$ be the prime ideal in $R_{o}[X]=R_{o}\left[X, \cdots, X_{n}\right]$ such that $R=R_{0}[X] / \mathscr{B}$. Then $\mathfrak{B} \cdot K[X] \cap R_{0}[X]=\mathfrak{B}$ and $\mathfrak{A}=$ $\mathfrak{B} \cap k[X]$. Since $K$ and $k(x)$ are linearly disjoint over $k$, it is well known that $\mathfrak{U} \cdot K[X]=\mathfrak{B} \cdot K[X]$ and $\mathfrak{U} \cdot R_{0}[X]=\mathfrak{B},[4$; Corollary 1, p. 67]. We shall use $\mathfrak{B}$ in both $R_{0}[X]$ and $K[X]$ as the prime ideal determined by $(x)=\left(x_{1}, \cdots, x_{n}\right)$. Since $R_{b} \otimes_{k} k[X]=R_{0}[X], \quad$ it follows that $R_{b} \otimes_{k} k[x]=R_{b}[x]$, i.e. $R_{b} \otimes_{k} k[x]$ is an integral domain. It follows from [6; Theorem 8, p. 400] that $R_{0}[x]$ is normal. Conversely if $R_{0}[x]$ is normal, then $R_{0}[x]_{\mathrm{p}}$ is normal for each $\mathbf{p} \in \operatorname{Spec}\left(R_{0}[x]\right)$. Let $\mathbf{p}^{c}=$ $\mathbf{p} \cap k[x]$ for $\mathbf{p} \in \operatorname{Spec}\left(R_{b}[x]\right)$ and $\mathbf{p} \cap R_{\boldsymbol{b}}=\{0\}$. Then $k[x]_{\mathbf{p}}$ is also normal. Indeed let $\xi \in k(x)$ be integral over $k[x]_{\mathrm{p}^{\mathrm{c}}}$. Since $k[x]_{\mathrm{p}^{\mathrm{c}}} \subset$ $R_{o}[x]_{\mathrm{p}}$, therefore $\xi \in R_{0}[x]_{\mathrm{p}}$. Thus $\xi \in R_{0}[x]_{\mathrm{p}} \cap k(x)$. It is sufficient to show that $R_{b}[x]_{\mathrm{p}} \cap k(x) \subset k[x]_{\mathrm{p}}$. Let $S=R_{0}-\{0\}$. $K[x]=S^{-1} R_{0}[x]$ and 
$S^{-1} \mathbf{p}$ is a prime ideal in $K[x] . S^{-1} \mathbf{p} \cap k[x]=\mathbf{p} \cap k[x]$. Since $K$ and $k(x)$ are linearly disjoint over $k$, it follows from [4; Proposition 6, p. 92] that $K[x]_{s^{-1} \mathrm{p}} \cap k(x)=k[x]_{\mathrm{p}^{c}}$. Thus $k[x]_{\mathrm{p}^{\mathrm{c}}} \supset R_{0}[x]_{\mathrm{p}} \cap k(x)$, and $k[x]_{\mathrm{p}^{\mathrm{c}}}=$ $R_{0}[x]_{\mathrm{p}} \cap k(x)$. So $\xi \in k[x]_{\mathrm{p}^{\mathrm{c}}}$ and $k[x]_{\mathrm{p}^{\mathrm{c}}}$ is therefore normal.

We shall finish the proof by showing that $\operatorname{Spec}(k[x])=$ $\left\{\mathbf{p} \cap k[x] \mid \mathbf{p} \in \operatorname{Spec}\left(R_{0}[x]\right)\right.$ and $\left.\mathbf{p} \cap R_{0}=0\right\}$. Let $\mathbf{q}_{\mathscr{x}}$ be a prime ideal. There exists a prime ideal $Q_{\mathscr{x}}$ in $K[X]$ such that $Q_{\mathscr{X}} \cap k[X]=\mathbf{q}_{\mathscr{x}}$. Indeed, using Zariski's terminology [10; pp. 21-22 and pp. 161-176], we consider an algebraically closed field $\Omega$ containing $K$ and $\Omega$ is of infinite transcendence degree over $K$. Let $A_{n}^{\Omega}$ be the $n$ dimensional affine space, i.e. $A_{n}^{\Omega}=\left\{\left(a_{1}, \cdots, a_{n}\right) \mid a_{1}, \cdots, a_{n} \in \Omega\right\}$. Every prime ideal $P$ in $K[X]$ defines an irreducible algebraic variety $V$ over $K$ in $A_{n}^{\Omega}$. Every irreducible algebraic variety $V$ over $K$ carries a generic point $(\xi)=$ $\left(\xi_{1}, \cdots, \xi_{n}\right) \in A_{n}^{\Omega}$ over $K$, and $P=\{g(X) \in K[X] \mid g(\xi)=0\}$. Let $(\eta)=$ $\left(\eta_{1}, \cdots, \eta_{n}\right) \in A_{n}^{\Omega}$ be a generic point of $\mathbf{q}_{\mathscr{x}}$ over $k$, i.e. $\mathbf{q}_{\mathscr{x}}=$ $\{f(X) \in k[X] \mid f(\eta)=0\}$. Let $Q_{x}=\{F(X) \in K[X] \mid F(\eta)=0\}$. Then $Q_{\mathscr{x}}$ is a prime ideal and $Q_{\mathscr{x}} \cap k[X]=\mathbf{q}_{\mathscr{x}}$. Let $Q_{\mathscr{X}}^{\prime}=Q_{\mathscr{X}} \cap R_{\boldsymbol{o}}[X], Q_{\mathscr{x}}^{\prime} \cap R_{\boldsymbol{o}}=0$ and $Q_{\mathscr{x}}^{\prime} \cap k[X]=\mathbf{q}_{\mathscr{x}}$. Since $\mathfrak{A} \subset \mathbf{q}_{\mathscr{x}} \Leftrightarrow \mathfrak{B} \cdot K[X] \subset Q_{\mathscr{x}} \Leftrightarrow \mathfrak{B} \subset Q_{\mathscr{x}}^{\prime}$. Let $Q^{\prime}=Q_{x}^{\prime} / \mathcal{B} \subset R_{0}[x]$. Then $Q^{\prime} \cap k[x]=\mathbf{q}$. Thus each prime ideal in $k[x]$ is the contraction of a prime ideal in $R_{0}[x]$ intersecting $R_{b}$ at 0 .

As the assertion in the last part of the proof of the above theorem will be referred later, we would like to state it as a corollary.

COROLlary. Let $R_{0}$ be an integral domain containing a field $k$. Let $R=R_{0}\left[x_{1}, \cdots, x_{n}\right]$ be an integral domain finitely generated over $R_{0}$ as an algebra such that the quotient field $K$ of $R_{0}$ and the quotient field $k(x)$ of $k[x]=k\left[x_{1}, \cdots, x_{n}\right]$ are linearly disjoint over $k$. Then $\operatorname{Spec}(k[x])=$ $\left\{\mathbf{p} \cap k[x] \mid \mathbf{p} \in \operatorname{Spec}\left(R_{0}[x]\right)\right.$ and $\left.\mathbf{p} \cap R_{0}=0\right\}$. Moreover if $R$ is graded with $R_{0}$ as the component of homogeneous degree 0 , then $\operatorname{Proj}(k[x])=$ $\left\{\mathbf{p} \cap k[x] \mid \mathbf{p} \in \operatorname{Proj}\left(R_{0}[x]\right)\right\}=\{\mathbf{p} \cap k[x] \mid \mathbf{p} \in \operatorname{Proj} K[x]\}$.

Proof (of the last part). Let $\mathfrak{A}, \mathfrak{B}, \mathbf{q}, \mathbf{q}_{\mathscr{x}}$, and $Q_{\mathscr{x}}$ be the same as those in the proof of Theorem 3 . If $R$ is a graded domain, then both $\mathfrak{A}$ and $\mathfrak{B}$ are homogeneous ideals. If $\mathbf{q}$ is a nonirrelevant and homogeneous prime ideal in $k[x]$, then so is $\mathbf{q}_{x}$. Let $Q_{x}^{*}$ be the ideal in $K[x]$ generated by the homogeneous elements belonging to $Q_{\mathscr{x}}$. Then, by [10; Lemma 3, p. 153], $Q_{\mathscr{x}}^{*}$ is a prime ideal and clearly $Q_{\mathscr{x}}^{*} \cap k[X]=\mathbf{q}_{\mathscr{x}}$. Since $\mathbf{q}_{\mathscr{x}}$ is nonirrelevant, $Q_{\mathscr{x}}^{*}$ is also nonirrelevant, and $Q_{\mathscr{X}}^{*} \supset \mathfrak{B}$. Let $Q^{*}=Q_{\mathscr{x}}^{*} / \mathcal{B}$. We have $Q^{*} \cap k[x]=\mathbf{q}$. Therefore $\operatorname{Proj}(k[x])=$ $\left\{\mathbf{p} \cap k[x] \mid \mathbf{p} \in \operatorname{Proj}(R)\right.$ and $\left.\mathbf{p} \cap R_{\boldsymbol{o}}=0\right\}$.

Let us recall some definitions and facts: Let $R=\bigoplus_{i \geq 0} R_{i}$ be a graded integral domain. $\quad R$ is Noetherian if and only if $R_{b}$ is Noetherian and $R$ is an $R_{0}$-algebra of finite type. Let $\bar{R}$ be the integral closure of $R$ in its field of quotients $K$. Let $K_{i}$ be the homogeneous component of $K$ of 
degree $i$ as defined in $\S 2$. Then $\bar{R}$ is graded with $\bar{R}_{i}=\bar{R} \cap K_{i}$. Thus if $R$ is normal then $R_{0}$ must be normal.

Corresponding to Krull's characterization of a Noetherian domain being normal [7; (12.9), p. 41], we have the following theorem for normality of a Noetherian graded domain.

THEOREM 4. Let $R$ be a graded Noetherian domain such that $R_{1}-\mathbf{p} \neq \varnothing$ for each homogeneous prime ideal $\mathbf{p}$ of ht 1 in $R$. If (1) $R_{(\mathbf{p})}$ is normal for every homogeneous prime ideal $\mathbf{p}$ of height 1 and (2) the associated prime ideals of every nonzero homogeneous ideal are of height 1 , then $R$ is normal.

Proof. We first note that it follows from condition (1), Theorem 1 and Theorem 2 that $R_{\mathrm{p}}$ is normal for every $\mathbf{p} \in \operatorname{Spec}(R)$ and $h t(\mathbf{p})=1$. Let $K, \bar{R}$ and $\bar{R}_{i}$ be the same as defined in the preceeding. Let $\alpha \in \bar{R}$, $\alpha=\sum_{i=m}^{n} \alpha_{i}$ for some nonnegative integers $m$ and $n$ and $\alpha_{i} \in \bar{R}_{i}$. Let $\alpha_{i}=b_{i j} / a_{i l}$ where $j-l=i, b_{i j} \in R_{j}$ and $a_{i l} \in R_{l}$. If $a_{i l}$ is a unit in $R$ then $\alpha_{i} \in R$. If $a_{i l}$ is a nonunit, then the nonzero homogeneous principal ideal $\left(a_{i l}\right) R$ has a primary decomposition $\bigcap_{t=1}^{u} \mathbf{q}_{t}$ with $\mathbf{p}_{1}, \cdots, \mathbf{p}_{u}$ as the associated prime ideals. In view of $[\mathbf{1 0}$; Theorem 9 and Corollary; pp. 153-154] we may assume that $\mathbf{q}_{t}$ 's and $\mathbf{p}_{t}$ 's are homogeneous, (2) implies that $h t\left(\mathbf{p}_{t}\right)=1$ for $t=1,2, \cdots, u$. Thus $R_{\mathbf{p}_{t}}$ is normal for $t=$ $1,2, \cdots, u . \alpha_{i}$ is integral over $R$ implies that $\alpha_{i}$ is integral over $R_{\mathrm{p}_{t}}$ for $t=1,2, \cdots, u$. Hence $\alpha_{i} \in R_{\mathrm{p}_{i}}$ for $t=1,2, \cdots, u$. Therefore $b_{i j} \in$ $\bigcap_{t=1}^{u}\left(\left(a_{i l}\right) R_{\mathbf{p} t} \cap R\right)=\bigcap_{t=1}^{u} \mathbf{q}_{t}=\left(a_{i l}\right) R$. Thus $\alpha_{i}=b_{i j} / a_{i l} \in R$ and $\alpha=$ $\sum_{i=m}^{n} \alpha_{i} \in R . R$ is therefore normal.

Let $A=K\left[X_{1}, \cdots, X_{n}\right]$ be a polynomial ring over a field $K$. The smallest integer $d$ such that any chain of syzygies of the $A$-module $M$ terminates at $(d+1)$ th step is called the cohomological dimension of $M$ and is denoted by coh.d.(M). Let $\mathfrak{A} \subset A$ be a homogeneous ideal such that $\mathfrak{U} \neq(0), \neq(1)$. coh.d.( $\mathfrak{U}) \leqq n$ and it is $n$ if and only if $\left(X_{1}, \cdots, X_{n}\right) A$ is an associated prime ideal of $\mathfrak{A}$. Let $l$ be a form in $A$, and $l \notin K$. If $\mathfrak{A}: l=\mathfrak{A}$ then coh.d.( $\mathfrak{A}, l)=1+$ coh.d.( $\mathfrak{U})$.

THEOREM 5. Let $R=\bigoplus_{i \supseteq 0} R_{i}$ be a Noetherian graded integral domain generated over $R_{0}$ by nonzero homogeneous elements $x_{1}, \cdots, x_{n}$ of degree 1. Assume that $R_{0}$ contains a subfield $k$ over which $R_{0}$ and $k(x)=k\left(x_{1}, \cdots, x_{n}\right)$ are linearly disjoint and $R_{0}$ is normal. Assume tr.deg $k(x)>0$. Let $R_{o}[X]=R_{o}\left[X_{1}, \cdots, X_{n}\right]$ be the polynomial ring over $R_{g}$ in indeterminantes $X_{1}, \cdots, X_{n}$ and let $\mathfrak{B}$ be the ideal such that $R_{0}[x] \cong R_{0}[X] / \mathfrak{B}$. Let $\mathfrak{A}=\mathfrak{B} \cap k[X]$, and let $S=R_{0}-\{0\}$.

(1) If, for each $\mathbf{p} \in \operatorname{Proj}\left(R_{0}[x]\right), \quad R_{0}[x]_{(\mathrm{p})}$ is normal and coh.d. $S^{-1} \mathfrak{B}<n-1$, then $k[x]$ is normal.

(2) If $R_{0}$ and $k(x)$ are both separable over $k$, and if $R_{0}[x]_{(\mathrm{p})}$ is normal 
for all $\mathbf{p} \in \operatorname{Proj}\left(R_{0}[x]\right)$, and coh.d. $S^{-1} \mathfrak{B}<n-1$ then $R_{0}[x]$ is normal.

(3) If $R_{(\mathbf{p})}$ is normal for each $\mathbf{p} \in \operatorname{Proj}(R)$ and if coh.d. $\mathscr{B} \cdot S^{-1} R_{o}[X]=n-1$ then $R_{0}[x]$ is not normal.

Proof. (1) Both $\mathfrak{A}$ and $\mathfrak{B}$ are homogeneous ideals, $k[x]$ is graded. As projective scheme $\operatorname{Proj}\left(R_{0}[x]\right) \cong \operatorname{Proj}\left(\left(S^{-1} R_{0}\right)[x]\right)$ [1, Prop. (2.4.7), p. 30]. Therefore $\left(S^{-1} R_{0}\right)[x]$ is locally normal, i.e. $\left(S^{-1} R_{0}\right)[x]_{(\mathrm{p})}$ is normal for each $\mathbf{p} \in \operatorname{Proj}\left(S^{-1} R_{0}[x]\right)$. Since tr.deg. $S^{-1} R_{0}[x]>0$. If coh.d. $S^{-1} \mathfrak{B}<$ $n-1$, by [9, Theorem 3, p. 619], $S^{-1} R_{0}[x]$ is normal. Therefore $S^{-1} R_{0}[x]_{\mathrm{p}}$ is normal for every $\mathrm{p} \in \operatorname{Spec}\left(S^{-1} R_{0}[x]\right)$. Since $\left(S^{-1} R_{0}\right)[x]_{\mathrm{p}} \cap$ $k(x)=k[x]_{\mathbf{p}^{c}}$ as shown in the preceeding, where $\mathbf{p}^{c}=\mathbf{p} \cap k[x] . k[x]_{\mathbf{p}^{c}}$ is normal. By the Corollary to Theorem 3, $\operatorname{Spec}(k[x])=$ $\left\{\mathbf{p}^{c} \mid \in \operatorname{Spec}\left(S^{-1} R_{\boldsymbol{o}}\right)[x]\right\}$, we have that $k[x]_{\mathbf{q}}$ is normal for every $\mathbf{q} \in$ $\operatorname{Spec}(k[x])$. Therefore $k[x]$ is normal.

(2) By (1), $k[x]$ is normal. $R_{\boldsymbol{b}}$ is normal. It follows from Theorem $3, R_{0}[x]$ is normal.

(3) If coh.d.B $\cdot S^{-1} R_{0}[X]=n-1$, then it is well known that for a form $l$ in $R_{0}[X]$ prime to $\mathfrak{B}$ i.e. $\mathfrak{B}: l=\mathfrak{B}$, coh.d. $(\mathfrak{B}, l) \cdot S^{-1} R_{0}[X]=n$. Therefore $(\mathfrak{B}, l) \cdot S^{-1} R_{0}[X]$ has $(X) \cdot S^{-1} R_{0}[X]$ as an associated prime ideal. Since $\operatorname{dim} \mathfrak{B} \cdot S^{-1} R_{0}[X]>0,(\mathfrak{B}, l) S^{-1} R_{0}[X]$ has an embedded associated prime. On the other hand, it is easy to see that $(X) S^{-1} R_{0}[X] \cap R_{0}[X]=(X) R_{0}[X]$. Therefore it follows from [5, Lemma $7 \mathrm{c}$, p. 50] that $(\mathfrak{B}, l) R_{0}[X]$ has $(X) R_{0}[X]$ as an embedded associated prime ideal. Let $(\bar{l}) R_{0}[X]=(\mathfrak{B}, l) R_{0}[X] / \mathscr{B}$. Therefore $(\bar{l}) R_{0}[x]$ is a principal homogeneous ideal having $(x) \cdot R_{0}[x]$ as an embedded associated prime ideal. It follows from Theorem 4 that $R$ is not normal.

4. Integral closure of a graded ring. In this section, we study a general graded ring, $R=\bigoplus_{i \geqq 0} R_{i}$. Let $F$ be the total quotient ring of $R$, and let $\bar{R}$ be the integral closure of $R$ in $F$. In case of a graded domain, the integral closure $\bar{R}$ of $R$ in its quotient field $K$ is again graded and $\bar{R}_{i}=\bar{R} \cap K_{i}$ for $i \geqq 0$. We investigate $\bar{R}$ when $R$ is not an integral domain. A ring $R$ is normal if $R_{\mathrm{p}}$ is an integral domain and integrally closed in its quotient field for each $\mathbf{p} \in \operatorname{Spec}(R)$.

Let $R=\bigoplus_{l \geqq 0} R_{i}$. Let $U$ be the set of all nonzero divisors of $R$. Let $F$ be the total quotient ring and let $F_{i}=\left\{r_{l} / u_{s} \mid r_{l} \in R_{l}\right.$, $\left.u_{j} \in R_{j} \cap U, l-j=i\right\}$. These are the notations going to be used in the sequel.

THEOREM 6. Assume $U \cap R_{1} \neq \varnothing$ and let $u_{1} \in U \cap R_{1}$. Then (1) the ring $\sum_{1 \in Z} F_{1}$ is a direct sum, and $\bigoplus_{1 \in Z} F_{t}=F_{0}\left[u_{1}, 1 / u_{1}\right], F=F_{0}\left[u_{1}\right]_{U}, u_{1}$ is algebraically independent over $F_{0}$, and $F_{i}=F_{0} \cdot u_{1}$ for all $i \in Z$. If $F_{0}$ is Noetherian then so is $F$. (2) $F_{0}$ is reduced, i.e. $F_{0}$ has no nonzero nilpotent element, if and only if $R$ is reduced. (3) If $R$ is reduced and $F_{0}$ is 
Noetherian, then $F_{0}\left[u_{1}\right]$ is integrally closed in $F$. (4) If $R$ is reduced and $F_{0}$ is Noetherian, then $\bar{R}$ is a graded subring of $\bigoplus_{i \in Z} F_{i}$.

Proof. (1) It follows from the definition of $F_{i}$ 's that each $F_{i}$ is an additive group and $F_{i} \cdot F_{j} \subset F_{i+j} . \sum_{i \in Z} F_{i}$ is a ring. Let $f_{k}+\cdots+f_{s} \in \sum_{i \in Z} F_{i}$. Suppose $f_{k}+\cdots+f_{s}=0$. Let $f_{m}=r_{l_{m}} / u_{j_{m}}$ where $l_{m}-j_{m}=m$ and $m=$ $k, \cdots, s$. Let $u=\prod_{m=k}^{s} u_{j_{m}}$. Then $u f_{k}+\cdots+u f_{s}=0$ in $R$, and $u f_{k}, \cdots, u f_{s}$ are homogeneous elements of distinct degrees. Therefore $u f_{k}=\cdots=$ $u f_{s}=0$. Thus $f_{k}=\cdots=f_{s}=0$, and the sum $\sum F_{i}$ is therefore a direct sum. Let $f_{k} \in F_{k}$. Then $f_{k} / u_{1}^{k} \in F_{0}$. Therefore $f_{k} \in F_{0} \cdot u_{1}^{k}$ and $F_{k}=F_{0} \cdot u_{1}^{k}$. Hence $\bigoplus_{i \in Z} F_{i}=F_{0}\left[u_{1}, 1 / u_{1}\right]$. For any $f \in F$,

$$
f=\left(f_{k}+\cdots+f_{s}\right) / u=\frac{1}{u}\left(\frac{f_{k}}{u_{1}^{k}} u_{1}^{k}+\cdots+\frac{f_{s}}{u_{1}^{s}} u_{1}^{s}\right) .
$$

Therefore $F=F_{0}\left[u_{1}, 1 / u_{1}\right]_{U}=F_{0}\left[u_{1}\right]_{U} \cdot u_{1}$ is algebraically independent over $F_{0}$. Indeed, let $a_{0} u_{1}^{n}+a_{1} u_{1}^{n-1}+\cdots+a_{n}=0$, where $a_{i} \in F_{0}$ and $a_{0} \neq 0$. Writing $a_{i}=r_{l_{i}} / u_{j_{i}}$ with $l_{i}-j_{i}=i$, we have $a_{i} u_{1}^{n-1} \in F_{n-i}$. Therefore $a_{i} u_{1}^{n-1}=0$, and $a_{i}=0$ for $i=0,1, \cdots, n$. Therefore $u_{1}$ is algebraically independent over $F_{0}$.

If $F_{0}$ is Noetherian, then so is $F_{0}\left[u_{1}\right]$. Now $F=F_{0}\left[u_{1}\right]_{U}$. Therefore $F$ is also Noetherian.

(2) It is obvious that $R$ is reduced implies that $F_{0}$ is reduced. Conversely, we note if $\left(x_{m} / u_{I}^{m}\right)^{n}=0$, then $x_{m}=0$. Also if $y_{m} \in R_{m}$ such that $y_{m}^{n}=0$ then $\left(y_{m} / u_{1}^{m}\right)=0$. Thus $y_{m}=0$. Now let $y$ be a nilpotent element in $R$. Write $y=\dot{y}_{k}+\cdots+y_{s}$. For some positive integer $b$, $y^{b}=\left(y_{k}+\cdots+y_{s}\right)^{b}=0$. Thus $y_{k}^{b}=0$ and then $\left(y_{k+1}+\cdots+y_{s}\right)^{b}=0$ and so on we get $y_{m}^{b}=y_{m+1}^{b}=\cdots=y_{s}^{b}=0$, so $y_{m}=\cdots=y_{s}=0$. Therefore $y=0$ and $R$ is reduced.

(3) $F_{0}$ is reduced. It follows from that $F=F_{0}\left[u_{1}\right]_{U}$ and that $u_{1}$ is transcendental over $F_{0}$, the nonzero divisors of $F_{0}$ are the same as the nonzero divisors of $R$ in $F_{0}$. Let $U_{0}$ be the set of all nonzero divisors of $F_{0}$. Let $u_{0} \in U_{0}$, then $u_{0}=r_{m} / u_{m}$ where $u_{m} \in U$ and $r_{m} \in R_{m}$. Moreover $r_{m} \in U$ also. Thus $u_{0}$ is a unit i.e. $U_{0}$ is a multiplicative group in $F_{0}$. Hence the total quotient ring $\left(F_{0}\right)_{U_{0}}=F_{0}$. Since $F_{0}$ is Noetherian and reduced, therefore, $F_{0}=\bigoplus_{i=1}^{S} G_{i}$ where $G_{i}$ 's are fields. It follows from [2; Proposition (6.5.2), p. 146] that $F_{0}$ is normal.

It follows from [5; Proposition (1.7.8), p. 116] that $F_{0}\left[u_{1}\right]$ is normal. Since $F_{0}\left[u_{1}\right]$ is a polynomial ring in $u_{1}$, and $F_{0}$ is reduced, therefore $F_{0}\left[u_{1}\right]$ is also reduced. $F_{0}$ is Noetherian implies that $F$ is Noetherian. Then $F=\bigoplus_{i=1}^{n} H_{i}$ where $H_{i}$ 's are fields. Thus it follows from [2; Proposition (6.5.2), p. 146] that $F_{0}\left[u_{1}\right]$ is integrally closed.

Note: Let $A=Z /(4)[X]$, the polynomial ring in $X$ over $Z /(4) . Z /(4)$ is integrally closed, while $A$ is not. Indeed, let $y=(x+1) /(x-1)$, $y^{2}-1=0, y \notin A$. 
(4) Let $x \in \bar{R}$. Since $R \subset R_{0}\left[u_{1}\right], x$ is integral over $F_{0}\left[u_{1}\right]$. By (3), $\bar{R} \subset F_{0}\left[u_{1}\right]$. The rest of the proof is practically the same argument used in the proof of [10; Theorem 11, p. 157]. We summarize the proof: Let $x \in \bar{R}, x=x_{k}+\cdots+x_{s}, k \leqq s, x_{k} \neq 0$ is called the initial homogeneous term. We want to show that each $x_{i}, i=k, \cdots, s$, is integral over $R$ also. Since $x \in \bar{R} \subset \sum F_{i}$, there exists $u_{m} \in R_{m} \cap U$ for some positive integer $m$, such that $u_{m} x \in R$. Case (a), if $R$ is Noetherian, then $R[x]$ is a finite $R$-module. There exists an integer $\lambda>0$ such that $u_{m}^{\lambda} x^{i} \in R$ for all integer $i \geqq 0$. Let $d=u_{m}^{\lambda}$. Then $d R[x] \subset R$. The initial homogeneous term $d x^{i}$ is $d x_{k}^{i} . d x^{i} \in R$ implies $d x_{k}^{i} \in R$. Therefore $x_{k}^{i} \in(1 / d) R$, a Noetherian $R$-module. Therefore $R\left[x_{k}\right] \subset R \cdot 1 / d$ is a Noetherian $R$ submodule. Therefore $x_{k}$ is integral over $R$. Repeating that argument to $x-x_{k}=x_{k+1}+\cdots+x_{s}$, we conclude that $x_{i} \in \bar{R}$ for $i=k, \cdots, s$. Therefore $\bar{R}$ is graded in this case. Next we look at case (b): $R$ is not Noetherian. Let $x \in \bar{R}$, and $x^{n}+a_{1} x^{n-1}+\cdots+a_{n}=0$ where $a_{1}, \cdots, a_{n} \in R$. As in case (a), there is a homogeneous nonzero divisor $d \in R$ such that $d x_{k}^{i} \in R$. Let $\left\{y_{1}, \cdots, y_{\mathcal{N}}\right\}=\left\{d, d x_{k}\right.$, and homogeneous components of $a_{\imath}$ 's $\}$. Let $A=k\left[y_{1}, \cdots, y_{\mathcal{N}}\right]$, where $k=Z$ or $Z /(n)$ according to whether $R$ is of characteristic 0 or $n>0$. $A \subset R$. Let $A_{q}=A \cap R_{q}$. Then $A=\sum A_{a}$ is a graded subring of $R$. $U \cap A$ contains $d$. Therefore $A_{U \cap A}$, the total quotient ring of $A$, contains $x_{k}$, and hence contains $x$ also. Thus the above integral relation takes place in $A_{U \cap A}$. Since $A$ is Noetherian, therefore case (a) is applicable. Therefore $x_{k}$ is integral over $A$. hence $x_{k}$ is integral over $R$.

\section{REFERENCES}

1. A. Grothendieck, J. Dieudonné, Eléments de Géométrie Algébrique II, IV Inst. Hautes Études Sci. Publ. Math. No. 8 (1961), No. 20 (1964).

2. ——, Eléments de Géométrie Algébrique I, Springer-Verlag, Berlin-Heidelberg-New York (1971).

3. R. Hartshorne, Introduction to Algebraic Geometry, Chapter III, Lecture Notes, University of California, Berkeley (1973).

4. S. Lang, Introduction to Algebraic Geometry, Interscience, New York (1964).

5. H. Matsumura, Commutative Algebra, W. A. Benjamin Inc., New York (1970).

6. M. Nagata, A general theory of algebraic geometry over Dedekind domains II, Amer. J. Math., 80 (1958), pp. 382-420.

7. - Local rings, Interscience, New York, 1962.

8. Y. Nakai, On the arithmetic normality of hyperplane sections of algebraic varieties, Memoirs of the College of Science, University of Kyoto, Series A, 29 (1955), 159-163, Corrections, 30 (1957), 201.

9. A. Seidenberg, The hyperplane sections of arithmetically normal varieties, Amer. J. Math., 94 (1972), 609-630.

10. O. Zariski, P. Sammuel, Commutative Algebra II, Van Nostrand, Princeton-Toronto-LondonNew York (1960). 



\section{PACIFIC JOURNAL OF MATHEMATICS}

\section{EDITORS}

RICHARD ARENS (Managing Editor)

University of California

Los Angeles, California 90024

\author{
R. A. Beaumont \\ University of Washington \\ Seattle, Washington 98105
}

\section{J. DugunduI}

Department of Mathematics University of Southern California Los Angeles, California 90007

D. Gilbarg and J. Milgram

Stanford University

Stanford, California 94305

\section{ASSOCIATE EDITORS}

E. F. BECKENBACH
B. H. NeumanN

F. Wolp
K. YoshidA

\section{SUPPORTING INSTITUTIONS}

UNIVERSITY OF BRITISH COLUMBIA

CALIFORNIA INSTITUTE OF TECHNOLOGY

UNIVERSITY OF CALIFORNIA

MONTANA STATE UNIVERSITY

UNIVERSITY OF NEVADA

NEW MEXICO STATE UNIVERSITY

OREGON STATE UNIVERSITY

UNIVERSITY OF OREGON

OSAKA UNIVERSITY

\author{
UNIVERSITY OF SOUTHERN CALIFORNIA \\ STANFORD UNIVERSITY \\ UNIVERSITY OF HAWAII \\ UNIVERSITY OF TOKYO \\ UNIVERSITY OF UTAH \\ WASHINGTON STATE UNIVERSITY \\ UNIVERSITY OF WASHINGTON \\ AMERICAN MATHEMATICAL SOCIETY
}

The Supporting Institutions listed above contribute to the cost of publication of this Journal, but they are not owners or publishers and have no responsibility for its contents or policies.

Mathematical papers intended for publication in the Pacific Journal of Mathematics should be in typed form or offset-reproduced (not dittoed). double spaced with large margins. Underline Greek letters in red, German in green, and script in blue. The first paragraph or two must be capable of being used separately as a synopsis of the entire paper. Items of the biblography should not be cited there unless absolutely necessary, in which case they must be identified by author and Journal, rather than by item number. Manuscripts, in duplicate, may be sent to any one of the four editors. Please classify according to the scheme of Math. Reviews, Index to Vol. 39. All other communications should be addressed to the managing editor, or Elaine Barth, University of California, Los Angeles, California, 90024.

100 reprints are provided free for each article, only if page charges have been substantially paid. Additional copies may be obtained at cost in multiples of 50 .

The Pacific Journal of Mathematics is issued monthly as of January 1966. Regular subscription rate: $\$ 72.00$ a year (6 Vols., 12 issues). Special rate: $\$ 36.00$ a year to individual members of supporting institutions.

Subscriptions, orders for back numbers, and changes of address should be sent to Pacific Journal of Mathematics, 103 Highland Boulevard, Berkeley, California, 94708.

PUBLISHED BY PACIFIC JOURNAL OF MATHEMATICS, A NON-PROFIT CORPORATION Printed at Jerusalem Academic Press, POB 2390, Jerusalem, Israel.

Copyright (C) 1976 Pacific Journal of Mathematics All Rights Reserved 


\section{Pacific Journal of Mathematics}

\section{Vol. 64, No. $2 \quad$ June, 1976}

Richard Fairbanks Arnold and A. P. Morse, Plus and times............. 297

Edwin Ogilvie Buchman and F. A. Valentine, External visibility ......... 333

R. A. Czerwinski, Bonded quadratic division algebras.............. 341

William Richard Emerson, Averaging strongly subadditive set functions in unimodular amenable groups. II .........................

Lynn Harry Erbe, Existence of oscillatory solutions and asymptotic behavior for a class of third order linear differential equations ............. 369

Kenneth R. Goodearl, Power-cancellation of groups and modules........ 387

J. C. Hankins and Roy Martin Rakestraw, The extremal structure of locally compact convex sets ...................................

Burrell Washington Helton, The solution of a Stieltjes-Volterra integral

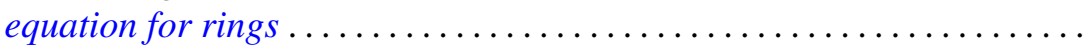

Frank Kwang-Ming Hwang and Shen Lin, Construction of 2-balanced

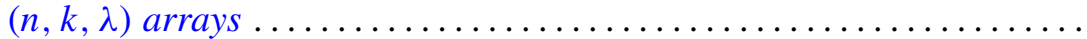

Wei-Eihn Kuan, Some results on normality of a graded ring ... 455

Dieter Landers and Lothar Rogge, Relations between convergence of series and convergence of sequences ......................... 465

Lawrence Louis Larmore and Robert David Rigdon, Enumerating immersions and embeddings of projective spaces ................

Douglas C. McMahon, On the role of an abelian phase group in relativized problems in topological dynamics..................

Robert Wilmer Miller, Finitely generated projective modules and TTF classes...

Yashaswini Deval Mittal, A class of isotropic covariance functions ...

Anthony G. Mucci, Another martingale convergence theorem ...

Joan Kathryn Plastiras, Quasitriangular operator algebras ...

John Robert Quine, Jr., The geometry of $p\left(S^{1}\right) \ldots \ldots \ldots$. 